\section{Genetic evolution of in situ follicular neoplasia to aggressive B-cell lymphoma of germinal center subtype}

Antonio Vogelsberg, ${ }^{1}$ Julia Steinhilber, ${ }^{1}$ Barbara Mankel, ${ }^{1}$ Birgit Federmann, ${ }^{1}$ Janine Schmidt, ${ }^{1}$ Ivonne A. Montes-Mojarro, ${ }^{1}$ Katrin Hüttl, ${ }^{2}$ Maria RodriguezPinilla, ${ }^{3}$ Praveen Baskaran, ${ }^{4}$ Sven Nahnsen, ${ }^{4}$ Miguel A. Piris, ${ }^{3}$ German Ott, ${ }^{2}$ Leticia Quintanilla-Martinez, ${ }^{1}$ Irina Bonzheim, ${ }^{1 /}$ and Falko Fend ${ }^{11}$

${ }^{1}$ Institute of Pathology and Neuropathology, University Hospital and Comprehensive Cancer Center Tübingen, Tübingen, Germany; ${ }^{2}$ Department of Clinical Pathology, RobertBosch-Krankenhaus, and Dr. Margarete Fischer-Bosch Institute of Clinical Pharmacology, Stuttgart, Germany; ${ }^{3}$ Department of Pathology, Fundación Jiménez Díaz, Madrid, Spain and ${ }^{4}$ Quantitative Biology Center, University of Tübingen, Tübingen, Germany

"IB and FF contributed equally as co-senior authors.

\section{ABSTRACT}

Tn situ follicular neoplasia (ISFN) is the earliest morphologically identifiable precursor of follicular lymphoma (FL). Although it is genetiL cally less complex than FL and has low risk for progression, ISFN already harbors secondary genetic alterations, in addition to the defining $\mathrm{t}(14 ; 18)$ (q32; $\mathrm{q} 21)$ translocation. $\mathrm{FL}$, in turn, frequently progresses to diffuse large B-cell lymphoma (DLBCL) or high-grade B-cell lymphoma (HGBL). By BCL2 staining of available reactive lymphoid tissue obtained at any time point in patients with aggressive B-cell lymphoma (BCL), we identified ten paired cases of ISFN and DLBCL/HGBL, including six de novo tumors and four tumors transformed from FL as an intermediate step, and investigated their clonal evolution using microdissection and next-generation sequencing. A clonal relationship between ISFN and aggressive BCL was established by immunoglobulin and/or $B C L 2$ rearrangements and/or the demonstration of shared somatic mutations for all ten cases. Targeted sequencing revealed CREBBP, KMT2D, EZH2, TNFRSF14 and BCL2 as the genes most frequently mutated already in ISFN. Based on the distribution of private and shared mutations, two patterns of clonal evolution were evident. In most cases, the aggressive lymphoma, ISFN and, when present, FL revealed divergent evolution from a common progenitor, whereas linear evolution with sequential accumulation of mutations was less frequent. In conclusion, we demonstrate for the first time that $\mathrm{t}(14 ; 18)+$ aggressive $\mathrm{BCL}$ can arise from ISFN without clinically evident FL as an intermediate step and that during this progression, branched evolution is common.

\section{Introduction}

B-cell lymphomas (BCL) are thought to arise from premalignant precursor cells by stepwise accumulation of mutations fostering survival and clonal expansion. Whereas some premalignant lesions such as monoclonal gammopathy of unknown significance (MGUS) and monoclonal B-cell lymphocytosis (MBL) have been known for many years, there are no known precursors for de novo aggressive BCL. Diffuse large B-cell lymphoma (DLBCL) not otherwise specified (NOS) is the most frequent form of $\mathrm{BCL}$ and represents $25-35 \%$ of adult $\mathrm{BCL}$ in the Western world. Based on gene expression profiling DLBCL can be sub-classified into activated B-cell-like $(A B C)$ and germinal center B-cell-like (GCB) subtypes. ${ }^{1,2}$ Approximately $20-30 \%$ of DLBCL, mostly of GCB subtype, exhibit the $\mathrm{t}(14 ; 18)(\mathrm{q} 32 ; \mathrm{q} 21)$ translocation, the hallmark of follicular lymphoma (FL). ${ }^{3}$ This translocation causes constitutive overexpression of the anti-apoptotic protein
Ferrata Storti Foundation

Haematologica 2021

Volume 106(10):2673-2681

\section{Correspondence:}

FALKO FEND

falko.fend@med.uni-tuebingen.de

IRINA BONZHEIM

irina.bonzheim@med.uni-tuebingen.de

Received: April 8, 2020

Accepted: August 12, 2020.

Pre-published: August 27, 2020.

https://doi.org/10.3324/haematol.2020.254854

(C)2021 Ferrata Storti Foundation

Material published in Haematologica is covered by copyright. All rights are reserved to the Ferrata Storti Foundation. Use of published material is allowed under the following terms and conditions:

https://creativecommons.org/licenses/by-nc/4.0/legalcode. Copies of published material are allowed for personal or internal use. Sharing published material for non-commercial purposes is subject to the following conditions:

https://creativecommons.org/licenses/by-nc/4.0/legalcode, sect. 3. Reproducing and sharing published material for commercial purposes is not allowed without permission in writing from the publisher. 
BCL2 and effectively abrogates negative selection in the germinal center (GC), leading to prolonged survival in the GC environment. ${ }^{4 \cdot 6}$ Another category of aggressive BCL harboring $B C L 2$ (18q21) translocations are high-grade $\mathrm{B}$ cell lymphomas (HGBL) with an additional MYC rearrangement, so-called double-hit $(\mathrm{DH})$ or triple-hit (TH) lymphomas, when also carrying BCL6 translocations. ${ }^{3}$ In addition to de novo presentation, both DLBCL and HGBL can arise from indolent BCL, most commonly FL. ${ }^{3}$ Transformation of FL into an aggressive lymphoma occurs in $2-3 \%$ of patients per year and usually results in a GCB phenotype. ${ }^{3,7}$

The $t(14 ; 18)$ (q32;q21) occurs during early B-cell development and is considered a founding alteration. ${ }^{4}$ However, this translocation alone is insufficient to cause the development of $\mathrm{FL}$, as $\mathrm{t}(14 ; 18)+\mathrm{B}$ cells can be identified at low frequencies in the peripheral blood of about half of otherwise healthy adults over the age of $50 ., 3$ Distinct clones of these $t(14 ; 18)$-carrying cells, termed follicular lymphoma like B cells (FLLC), have been shown to persist and even expand over years without progressing to manifest FL in most individuals. ${ }^{3,9}$ The risk for progression depends on the clone size, rather than on the number of different $t(14 ; 18)+$ clones. ${ }^{10}$ The earliest identifiable tissue-based precursor of FL is in situ follicular neoplasia (ISFN), defined as colonization of GC by a monoclonal population of $\mathrm{t}(14 ; 18)+\mathrm{B}$ cells in otherwise reactive lymphoid tissues.,11 Although by definition the normal lymphoid architecture is not altered, ISFN can be identified immunohistochemically by virtue of its strong staining for BCL2 and CD10 and a low proliferation index. ${ }^{11}$ ISFN can occur syn- or metachronously with FL, as well as with other BCL, but can also be found in individuals without history of lymphoma. ${ }^{12,13}$ The risk of progression seems to be low. ${ }^{12}$ Although ISFN is considered a precursor lesion, it already demonstrates secondary genetic alterations typically associated with manifest FL, especially affecting chromatin modifier genes such as $C R E B B P$, and less frequently KMT2D and EZH2, known as important factors in FL pathogenesis. ${ }^{1416}$ Importantly, both ISFN and FLLC also exhibit persistent expression of activation-induced cytidine deaminase (AID), which catalyzes class switch recombination and somatic hypermutation (SHM). ${ }^{17,18}$ AID activity is responsible for the intraclonal heterogeneity of the re-arranged immunoglobulin heavy chain (IGH) genes and the acquisition of novel Nglycosylation sites in the IG variable regions. ${ }^{18,19}$ Novel glycosylation motifs are a feature frequently observed in $\mathrm{FL}$, but less so in normal B cells or other BCL, and are thought to enable the interaction with mannose-binding lectins, eliminating the need for conventional B-cell receptor signaling through antigen binding. ${ }^{18,20}$ Furthermore, AID activity is believed to be an important driver for the genetic evolution of FL, leading to increased genomic instability and the accumulation of additional aberrations. ${ }^{21,22}$

Given the well-known role of ISFN as clonally related premalignant FL precursor and the frequent occurrence of the $t(14 ; 18)$ translocation in both de novo and secondary DLBCL and HGBL, ${ }^{3,14,2,3,24}$ we aimed to identify syn- or metachronous ISFN in patients with DLBCL and HGBL, and used these paired samples to investigate the clonal relationship, clonal evolution and underlying genetic changes driving progression from ISFN to aggressive BCL.

\section{Methods}

\section{Sample selection}

Suitable cases were identified by searching the archives of the Institutes of Pathology of Tuebingen University Hospital and the Robert-Bosch-Krankenhaus (Stuttgart, Germany) for patients with a diagnosis of DLBCL or HGBL, with or without antecedent FL, for which reactive lymphoid tissue from any time point was available, and staining the lymphoid tissues for BCL2 to identify ISFN (as detailed in the Online Supplementary Appendix). The ISFN of case 8 has already been included in previous studies. ${ }^{14,15}$ An additional case was provided by the Hospital Universitario Fundación Jiménez Díaz (Madrid, Spain). All diagnoses were made according to the criteria of the 2017 World Health Organization classification and reviewed by two experienced hematopathologists (LQ-M and FF). ${ }^{3}$ This study was approved by the Ethics Committee of the University of Tuebingen (096/2016/B02).

\section{Microdissection, immunohistochemistry and fluorescence in situ hybridization}

Microdissection of ISFN samples was performed on $5 \mu \mathrm{m}$ hematoxylin and eosin stained formalin-fixed, paraffin-embedded (FFPE) sections with an Axiovert 200M microscope (Zeiss, Oberkochen, Germany) and the P.A.L.M. system (Palm@Robo software 3.0; Zeiss). Fluorescence in situ hybridization (FISH) was performed on FFPE sections using Vysis LSI BCL2, BCL6 and MYC Dual Color Break Apart Rearrangement Probes (Abbott Molecular, Wiesbaden, Germany). For additional information, including DNA extraction and immunohistochemistry, see the Online Supplementary Appendix.

\section{Polymerase chain reaction and sequencing of the $t(14 ; 18)$ breakpoint region}

The $t(14 ; 18)$ breakpoint regions were amplified by polymerase chain reaction (PCR) and sequenced using major breakpoint, minor cluster and intermediate cluster region primers together with a joining region consensus primer as previously described, as well as the IdentiClone BCL2/JH Translocation Assay (Invivoscribe, San Diego, CA, USA) (Online Supplementary Appendix).

\section{Clonality analysis and immunoglobulin sequencing}

Detection of monoclonal IGH and IGk light chain (IGK) gene rearrangements was performed using BIOMED-2 primers as previously described. ${ }^{25}$ Next-generation sequencing (NGS) of IGH genes was accomplished with the LymphoTrack Dx IGH Assay - PGM (Invivoscribe) on the Ion Torrent Personal Genome Machine (PGM; Thermo Fisher Scientific, Waltham, MA, USA). Data were analyzed with the LymphoTrack Dx Software - PGM (Invivoscribe). For a description of the IG sequence analysis and the construction of phylogenetic trees to illustrate the clonal evolution of the IGH, see the Online Supplementary Appendix.

\section{Targeted next-generation sequencing analysis}

Samples were subjected to NGS on the Ion Torrent PGM using AmpliSeq Custom Panels created with the Ion AmpliSeq Designer (Thermo Fisher Scientific). The panels target recurrent mutations of $\mathrm{FL}$ and DLBCL, covering $95.21 \%$ of the coding sequence of $B C L 2, B C L 6, B T G 1 / 2, C A R D 11, C D 79 B, C R E B B P$, EP300, FOXO1, GNA13, HIST1H1B-E, IGLL5, KMT2D, IRF4, MEF2B, PIM1, PRDM1, TBL1XR1, TNFAIP3, and TNFRSF14 as well as exons 2-5 of MYD88 and the Y646 EZH2 hotspot (Online Supplementary Table S1). In addition, all aggressive BCL were analyzed with the Ion Ampliseq TP53 Panel (Thermo Fisher 
Scientific). Variant validation was performed using either a bidirectional single amplicon resequencing approach or, for ISFN samples, a second targeted NGS analysis after microdissection of affected GC. For a detailed description of library preparation, sequencing, data analyses and validation, including a primer list, see the Online Supplementary Appendix and the Online Supplementary Table S2.

\section{Results}

\section{Clinical features}

Ten cases of aggressive BCL and paired ISFN were included (Table 1). In six cases, the aggressive lymphoma was considered de novo, whereas four cases also had an associated FL. In two of the four latter cases, the FL component was only detected during the screening of lymphoid tissues originally interpreted as reactive, while the two others had a history of FL. None of the six de novo cases developed or relapsed as FL during follow-up, which ranged from 7 to 54 months. In seven of ten cases, the ISFN lesions were present simultaneously in seemingly non-involved lymph nodes (LN) adjacent to the aggres- sive component. In cases 4,5 , and 7 , the ISFN was identified retrospectively in $\mathrm{LN}$ resected for other reasons several years prior to the DLBCL diagnosis.

\section{Histological and immunohistochemical findings}

The ISFN samples exhibited the typical features with overall preserved LN architecture and involved GC showing strong staining for BCL2 and CD10 and a very low proliferation rate (Figure $1 \mathrm{~A}$ and $\mathrm{B}$ ). All aggressive $\mathrm{BCL}$, including eight DLBCL (one case with two samples) and three DH/TH HGBL, were classified as GCB subtype according to the Hans algorithm and expressed BCL2 (Table 2; Figure 1D). The aggressive tumors of cases 1 and 3 exhibited strong and homogenous P53 expression, whereas the DLBCL of case 2 was completely negative. All of these samples showed TP53 mutations (Figure 2; Online Supplementary Table S4). All FL were grade 1/2 and expressed BCL2. A summary of immunohistochemical findings is included in the Online Supplementary Table S3.

\section{Chromosomal translocations}

FISH analysis with a BCL2 break-apart probe confirmed a break in BCL2 for all samples of nine cases, indicative of

Table 1. Clinical data of patients with in situ follicular neoplasia and aggressive B-cell lymphoma.

\begin{tabular}{|c|c|c|c|c|c|c|c|}
\hline Patient & $\begin{array}{l}\text { Age* } \\
\text { (years) }\end{array}$ & Sex & Diagnosis & Site & Stage & Additional information & Follow-up (Ireatment) \\
\hline \multicolumn{8}{|c|}{ De novo aggressive B-cell lymphoma } \\
\hline 1 & 59 & M & $\begin{array}{l}\text { ISFN } \\
\text { HGBL-TH }\end{array}$ & $\begin{array}{l}\text { Mesenterial LN } \\
\text { Mesenterial LN }\end{array}$ & IVA/IE & Synchronous with HGBL & $\mathrm{CR}-7$ months $(\mathrm{Sx}+\mathrm{CT})$ \\
\hline 2 & 61 & $\mathrm{~F}$ & $\begin{array}{l}\text { ISFN } \\
\text { DLBCL }\end{array}$ & $\begin{array}{l}\text { Mesenterial LN } \\
\text { Mesenterial LN }\end{array}$ & N/A & Synchronous with DLBCL & N/A \\
\hline 3 & 64 & M & $\begin{array}{l}\text { ISFN } \\
\text { DLBCL } \\
\text { DLBCL }\end{array}$ & $\begin{array}{l}\text { Axillary LN } \\
\text { Axillary LN } \\
\text { Cervical LN }\end{array}$ & IIIA & $\begin{array}{l}\text { Synchronous with primary DLBCL } \\
\text { Primary DLBCL } \\
7 \text { months after primary DLBCL }\end{array}$ & DOD (CT+ RT) \\
\hline 4 & 76 & $\mathrm{~F}$ & $\begin{array}{l}\text { ISFN } \\
\text { DLBCL }\end{array}$ & $\begin{array}{l}\text { Mesenterial LN } \\
\text { Paravertebral }\end{array}$ & IIA & $\begin{array}{l}\text { Resected during surgery for } \\
\text { colorectal cancer } 159 \text { months prior } \\
\text { to DLBCL diagnosis }\end{array}$ & CR - 54 months (CT) \\
\hline 5 & 67 & M & $\begin{array}{l}\text { ISFN } \\
\text { DLBCL }\end{array}$ & Mesenterial LN & IIIA & $\begin{array}{l}\text { Resected during surgery for bowel } \\
\text { obstruction } 42 \text { months prior to DLBCL } \\
\text { diagnosis }\end{array}$ & CR - 38 months (CT) \\
\hline 6 & 74 & $\mathrm{~F}$ & $\begin{array}{l}\text { ISFN } \\
\text { DLBCL }\end{array}$ & $\begin{array}{l}\text { Mesenterial LN } \\
\text { Small intestine }\end{array}$ & N/A & Synchronous with DLBCL & N/A \\
\hline
\end{tabular}

\section{Transformed FL}

$7 \quad 54$

$54 \quad \mathrm{~F}$

F ISFN

Axillary LN

\begin{tabular}{lllll} 
& & & FL & Cervical LN \\
& & & DLBCL & Cervical LN \\
$8^{\sharp}$ & \multirow{2}{*}{74} & F & ISFN & Mesenterial LN \\
& & & FL & Mesenterial LN \\
& & & HGBL-DH & Small intestine \\
& & & ISFN & Mesenterial LN \\
& & & FL & Mesenterial LN \\
& 64 & & HGBL-DH & Large intestine \\
& & & ISFN & Mesenterial LN \\
10 & 54 & M & FL & Mesenterial LN \\
& & & DLBCL & Mesenterial LN
\end{tabular}

Sentinel node for melanoma

116 months prior to DLBCL diagnosis

Synchronous with DLBCL, Grade 1/2

IIIS

Synchronous with HGBL

Synchronous with HGBL, Grade 1/2

IIA

Synchronous with HGBL

Synchronous with HGBL, Grade 1/2

Synchronous with DLBCL

Synchronous with DLBCL, Grade 1/2
CR - 54 months (CT)

CR - 90 months $(\mathrm{Sx}+\mathrm{CT})$

CR - 133 months $(\mathrm{Sx}+\mathrm{CT})$

CR - 158 months $(\mathrm{Sx}+\mathrm{CT})$

CR: complete remission; CT: chemotherapy; DOD: dead of disease; F: female; M: male; N/A: information not available; RT: radiotherapy; Sx: surgery; ISFN: in situ follicular neoplasia ;FL: follicular lymphoma; HGBL-TH: high-grade B-cell lymphoma triple-hit; HGBL-DH: HGBL double-hit; DLBCL: diffuse large B-cell lymphoma; LN: lymph node. *Age at diagnosis of aggressive B-cell lymphoma.\#FL not available for analysis. 


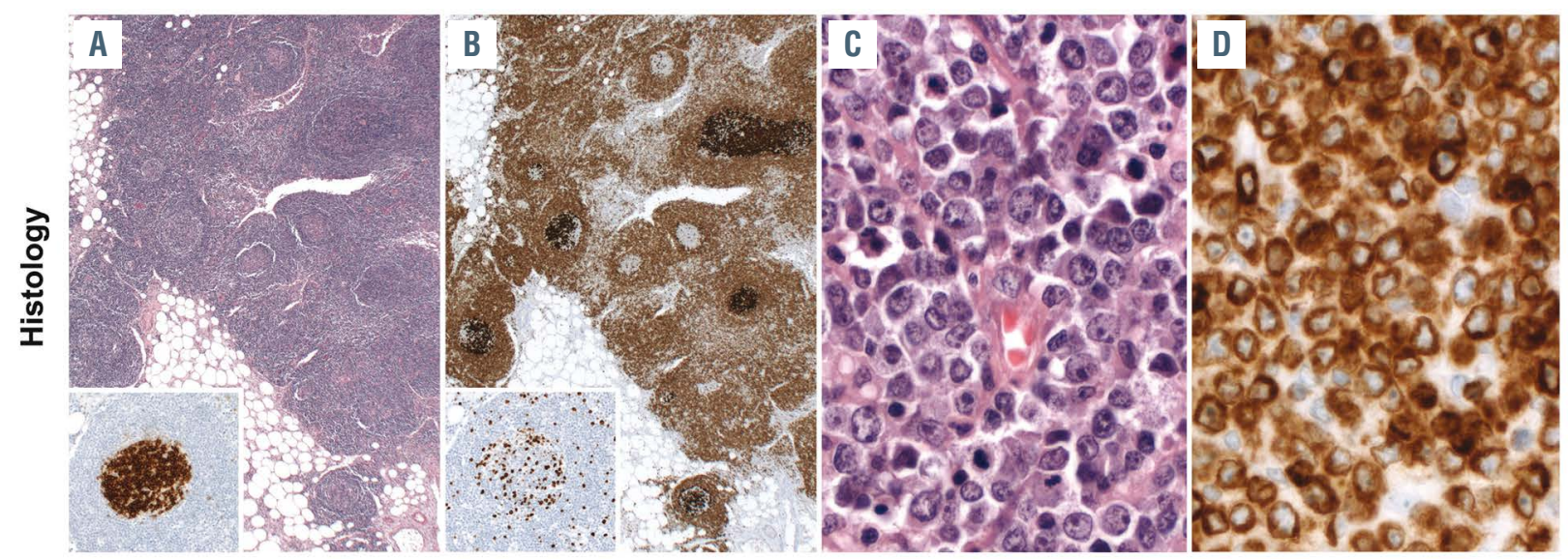

E

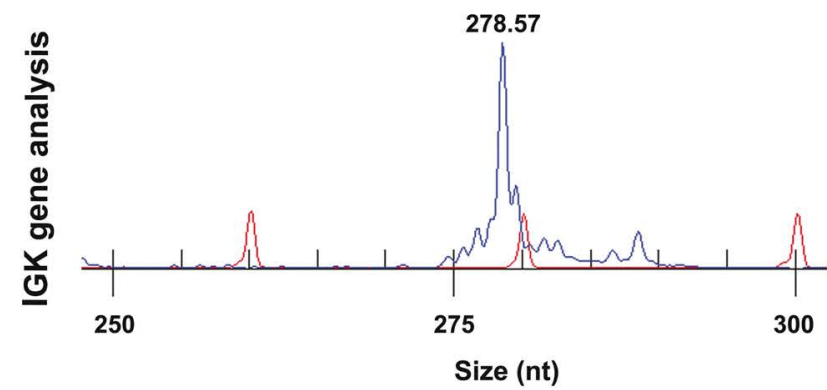

$\mathrm{F}$

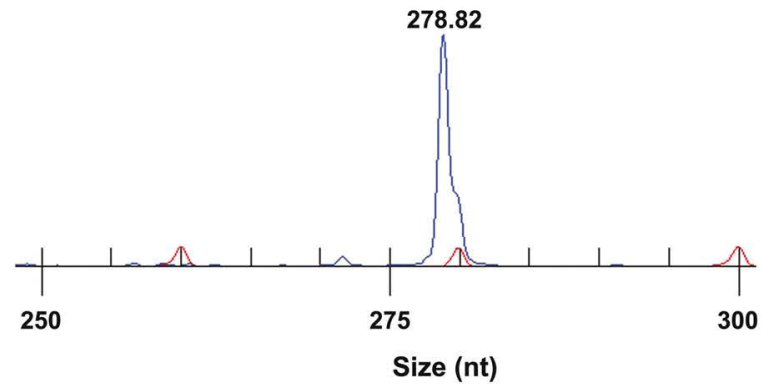

G 돈 de novo nucleotides

JH sequence (J6)

Figure 1. Morphology and molecular findings of the clonally related in situ follicular neoplasia and de novo high-grade B-cell lymphoma of case 1. (A) "Reactive" lymph node (LN) with in situ follicular neoplasia (ISFN) stained with hematoxylin and eosin (H\&E). Note the intact lymphoid architecture. Inset: higher magnification of a strongly CD10 positive germinal center (GC). Original magnification 25x and 100x. (B) Strong BCL2 expression in GC colonized by ISFN. Inset: MIB1 stain with higher magnification demonstrating a low proliferation index. Original magnification 25x and 100x. (C and D) LN biopsy depicting high-grade B-cell lymphoma (HGBL) stained with H\&E and BCL2. Original magnification 400x. (E and F) Immunoglobulin $\mathrm{K}$ light chain (IGK) GeneScan analysis demonstrating a rearrangement involving the $\mathrm{K}$ deleting element (Kde) with matching monoclonal peaks of 279 nucleotides ( $\mathrm{nt}$ ) in both lesions. (G) Sequencing of the BCL2 breakpoint revealed an identical $B C L 2 / \mathrm{JH}$ junction in both samples, confirming their clonal relationship.

the $t(14 ; 18)$ (q32; $q 21)$ translocation (Table 2). In case 5, $B C L 2$ and IGH break-apart probes did not demonstrate re-arrangements in either sample. However, using an IGH/BCL2 dual-color, double fusion probe, both ISFN and DLBCL showed an aberrant hybridization pattern with a single fusion signal, suggesting a cryptic BCL2 translocation. Amplification of the BCL2 breakpoint was successful for the samples of seven cases (cases 1, 3, 6, 7, 8,9 , and 10), with six breaks located in the major breakpoint region (MBR) and one (case 8) in the 3'MBR subcluster. Sequencing confirmed identical breakpoints for all paired samples (Figure 1G). MYC translocations were demonstrated in the aggressive component of three cases (cases 1, 8, and 9), with an additional break in BCL6 in case 1 , resulting in a diagnosis of $\mathrm{HGBL}$ with $\mathrm{DH}$ or $\mathrm{TH}$, respectively (Figure 2). The corresponding ISFN and FL lesions showed no alterations of $M Y C$ or BCL6.

\section{Clonality and immunoglobulin sequence analysis}

The results of the IG analysis are summarized in Table 2 and Figure 2. A clonal relationship based on IG rearrangements was demonstrated for seven of ten paired samples (cases 1, 2, 4, 7, 8, 9, and 10) by NGS of the IGH and/or by an identical clonal peak in IGH or IGK GeneScan analysis (Figure $1 \mathrm{E}$ and F). In case 10, the presence of a clonal IGH rearrangement in the ISFN was demonstrated by the use of clone-specific primers, which produced the same peak of 127 base pairs in the paired ISFN, FL and DLBCL lesions, confirming their clonal relationship (see Online Supplementary Appendix). In case 6, NGS demonstrated a clonal rearrangement in the DLBCL, but not in the corresponding ISFN, although both samples were shown to be clonally related by sequencing of their BCL2 breakpoint. Clone-specific primers designed for the DLBCL rearrangement also failed to amplify a specific product in the paired ISFN (see Online Supplementary Appendix). In contrast, cases 3 and 5 did not exhibit amplifiable clonal IG rearrangements in any of the samples. Thus, together with the results of the BCL2 breakpoint analysis, a clonal relationship between the ISFN and the corresponding lymphomas was firmly established for five of six de novo and four of four transformed cases.

Among samples successfully sequenced with the Lymphotrack Assay, we found novel N-glycosylation 
Table 2. Cell of origin, BCL2 translocation and immunoglobulin gene analysis.

\begin{tabular}{|c|c|c|c|c|c|c|c|c|c|}
\hline Case & Diagnosis & $\begin{array}{l}\text { Cell of origin based } \\
\text { on Hans algorithm }\end{array}$ & $\begin{array}{l}\text { BCL2 } \\
\text { FISH }\end{array}$ & $\begin{array}{c}\text { BCL2 } \\
\text { breakpoint }\end{array}$ & Clonality* & $\begin{array}{l}\text { IGH V/J } \\
\text { usage }\end{array}$ & \multicolumn{2}{|c|}{$\begin{array}{l}\text { Clycosylation site } \\
\text { Location Motif }\end{array}$} & Status \\
\hline \multicolumn{10}{|c|}{ De novo aggressive B-cell lymphoma } \\
\hline 1 & $\begin{array}{l}\text { ISFN } \\
\text { HGBL-TH }\end{array}$ & $\overline{\mathrm{GCB}}$ & $\begin{array}{l}+ \\
+\end{array}$ & $\begin{array}{l}\text { MBR-JH } \\
\text { MBR-JH }\end{array}$ & $\begin{array}{l}\text { Mono } \\
\text { Mono }\end{array}$ & $\begin{array}{l}\text { V3/J4 } \\
\text { V3/J4 }\end{array}$ & $\begin{array}{l}\text { CDR3 } \\
\text { CDR3 }\end{array}$ & $\begin{array}{l}\text { NLS } \\
\text { NLS }\end{array}$ & Clonally related \\
\hline 2 & $\begin{array}{l}\text { ISFN } \\
\text { DLBCL }\end{array}$ & $\overline{\mathrm{GCB}}$ & $\begin{array}{l}+ \\
+\end{array}$ & $\begin{array}{l}\text { Neg } \\
\text { Neg }\end{array}$ & $\begin{array}{l}\text { Mono } \\
\text { Mono }\end{array}$ & $\begin{array}{l}\mathrm{V} 2 / \mathrm{J} 4 \\
\mathrm{~V} 2 / \mathrm{J} 4\end{array}$ & $\begin{array}{l}\text { CDR3 } \\
\text { CDR3 }\end{array}$ & $\begin{array}{l}\text { NDS } \\
\text { NTS }\end{array}$ & Clonally related \\
\hline 3 & $\begin{array}{l}\text { ISFN } \\
\text { DLBCL } \\
\text { DLBCL }\end{array}$ & $\begin{array}{l}-\overline{\mathrm{GCB}} \\
\mathrm{GCB}\end{array}$ & $\begin{array}{l}+ \\
+ \\
+\end{array}$ & $\begin{array}{l}\text { MBR-JH }{ }^{\dagger} \\
\text { MBR-JH } \\
\text { MBR-JH }\end{array}$ & $\begin{array}{l}\text { Poly } \\
\text { Poly } \\
\text { Poly }\end{array}$ & - & \multicolumn{2}{|c|}{-} & Clonally related \\
\hline 4 & $\begin{array}{l}\text { ISFN } \\
\text { DLBCL }\end{array}$ & $\overline{\mathrm{GCB}}$ & $\begin{array}{l}+ \\
+\end{array}$ & $\begin{array}{l}\text { Neg } \\
\text { Neg }\end{array}$ & $\begin{array}{l}\text { Mono } \\
\text { Mono }\end{array}$ & $\begin{array}{l}\text { V3/J6 } \\
\text { V3/J6 }\end{array}$ & $\begin{array}{l}\text { CDR3 } \\
\text { CDR3 }\end{array}$ & $\begin{array}{l}\text { NAS } \\
\text { NAS }\end{array}$ & Clonally related \\
\hline 5 & $\begin{array}{l}\text { ISFN } \\
\text { DLBCL }\end{array}$ & $\overline{\mathrm{GCB}}$ & $\begin{array}{l}+^{\mathrm{f}} \\
+^{\mathrm{f}}\end{array}$ & $\begin{array}{l}\text { Neg } \\
\text { Neg }\end{array}$ & $\begin{array}{l}\text { Poly } \\
\text { Poly }\end{array}$ & - & \multicolumn{2}{|c|}{ - } & Clonally related $\S$ \\
\hline 6 & $\begin{array}{l}\text { ISFN } \\
\text { DLBCL }\end{array}$ & $\overline{\mathrm{GCB}}$ & $\begin{array}{l}+ \\
+\end{array}$ & $\begin{array}{l}\text { MBR-JH } \\
\text { MBR-JH }\end{array}$ & $\begin{array}{l}\text { Poly } \\
\text { Mono }\end{array}$ & $\overline{\mathrm{V} 3 / \mathrm{J} 3}$ & \multicolumn{2}{|c|}{ - } & Clonally related \\
\hline \multicolumn{10}{|c|}{ Transformed FL } \\
\hline 7 & $\begin{array}{l}\text { ISFN } \\
\text { FL } \\
\text { DLBCL }\end{array}$ & $\frac{-}{\mathrm{GCB}}$ & $\begin{array}{l}+ \\
+ \\
+\end{array}$ & $\begin{array}{l}\text { MBR-JH } \\
\text { MBR-JH } \\
\text { MBR-JH }\end{array}$ & $\begin{array}{l}\text { Mono } \\
\text { Mono } \\
\text { Mono }\end{array}$ & $\begin{array}{l}\text { V3/J6 } \\
\text { V3/J6 } \\
\text { V3/J6 }\end{array}$ & $\begin{array}{l}\text { CDR3 } \\
\text { CDR3 } \\
\text { CDR3 }\end{array}$ & $\begin{array}{l}\text { NLT } \\
\text { NLT } \\
\text { NLT }\end{array}$ & Clonally related \\
\hline 8 & $\begin{array}{l}\text { ISFN } \\
\text { HGBL-DH }\end{array}$ & $\overline{\mathrm{GCB}}$ & $\begin{array}{l}+ \\
+\end{array}$ & $\begin{array}{l}\text { 3'MBR-JH } \\
\text { 3'MBR-JH }\end{array}$ & $\begin{array}{l}\text { Mono } \\
\text { Mono }\end{array}$ & $\begin{array}{l}\text { V3/J3 } \\
\text { V3/J3 }\end{array}$ & $\begin{array}{l}\text { FR2/CL } \\
\text { None }\end{array}$ & $\begin{array}{r}\text { NIT } \\
\text { PCR) }\end{array}$ & Clonally related \\
\hline 9 & $\begin{array}{l}\text { ISFN } \\
\text { FL } \\
\text { HGBL-DH }\end{array}$ & $\frac{-}{\mathrm{GCB}}$ & $\begin{array}{l}+ \\
+ \\
+\end{array}$ & $\begin{array}{l}\text { MBR-JH }{ }^{\dagger} \\
\text { MBR-JH } \\
\text { MBR-JH }\end{array}$ & $\begin{array}{l}\text { Mono } \\
\text { Mono } \\
\text { Mono }\end{array}$ & $\begin{array}{l}\mathrm{V} 3 / \mathrm{J} 4 \\
\mathrm{~V} 3 / \mathrm{J} 4 \\
\mathrm{~V} 3 / \mathrm{J} 4\end{array}$ & $\begin{array}{l}\text { CDR3 } \\
\text { CDR3 } \\
\text { None }\end{array}$ & $\begin{array}{l}\text { NCS } \\
\text { NCS } \\
\text { PCR) }\end{array}$ & Clonally related \\
\hline 10 & $\begin{array}{l}\text { ISFN } \\
\text { FL } \\
\text { DLBCL }\end{array}$ & $\frac{-}{\mathrm{GCB}}$ & $\begin{array}{l}+ \\
+ \\
+\end{array}$ & $\begin{array}{l}\text { MBR-JH } \\
\text { MBR-JH } \\
\text { MBR-JH }\end{array}$ & $\begin{array}{l}\text { Mono" } \\
\text { Mono" } \\
\text { Mono" }\end{array}$ & $\begin{array}{l}\text { V1/J6 } \\
\text { V1/J6 } \\
\text { Vl/J6 }\end{array}$ & $\begin{array}{l}\text { CDR3 } \\
\text { CDR3 } \\
\text { CDR3 }\end{array}$ & $\begin{array}{l}\text { NFS } \\
\text { NYS } \\
\text { NYS }\end{array}$ & Clonally related \\
\hline
\end{tabular}

CDR: complementarity-determining region; FR: framework region; Neg: negative (i.e., no BCL2 rearrangement detected by polymerase chain reaction [PCR]);V/J: variable/joining gene segment; ISFN: in situ follicular neoplasia ; FL: follicular lymphoma; HGBL-TH: high-grade B-cell lymphoma triple-hit; HGBL-DH: HGBL double-hit; DLBCL: diffuse large B-cell lymphoma; LN: lymph node; MBR: major breakpoint region; GCB: germinal center B-cell-like; IGH: immunoglobulin H. *Based on Lymphotrack and/or GeneScan analysis

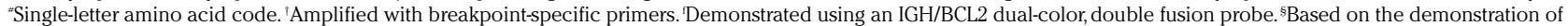
shared mutations."Demonstrated with clone-specific primers.

sites in seven of seven ISFN, three of three FL and six of eight aggressive BCL. In three cases (cases 1,4 , and 7), the ISFN and their transformed counterpart(s) demonstrated identical glycosylation sites, whereas two ISFN (cases 2 and 10) showed motifs at the same location, but with a different sequence than those exhibited by the clonally related manifest lymphomas. Moreover, two HGBL lacked N-glycosylation sites, although novel motifs were detected in the related ISFN (cases 8 and 9) and FL (case 9) lesions. Intraclonal heterogeneity of the clonal IGH rearrangement was present in all types of samples. However, heterogeneity was more pronounced in the precursor lesions, as evidenced by more evenly distributed subclones, whereas DLBCL and HGBL samples exhibited one or two subclones that were highly dominant. Phylogenetic trees constructed for five cases demonstrated separate clustering of ISFN and DLBCL/HGBL sequences indicative of divergent evolution (Online Supplementary Figure S1).

\section{Mutational analysis reveals distinct clonal evolution patterns}

$B C L 2$ was the most frequently mutated gene, with all samples harboring at least one non-synonymous, synonymous or 5'UTR mutation, although most samples, including seven of ten ISFN lesions, demonstrated several BCL2 mutations (Figure 2; Online Supplementary Tables S4 and S5). Other recurrently mutated genes were CREBBP (11 of 24 samples), KMT2D (11 samples), and EZH2 (ten sam- ples), as well as TNFRSF14, IGLL5, and GNA13. In ISFN lesions, mutations in chromatin modifying genes remained the most frequent alterations, with five samples showing a CREBBP mutation. In contrast, TP53 (four samples), CD79B (one sample) and HIST1H1B (one sample) were exclusively altered in the aggressive components. All TP53 mutations were located in the DNA binding domain, with variant allele frequencies ranging from $52 \%$ to $84 \%$, indicating a loss of the second allele. For two samples (cases 1 and 2), this was confirmed by FISH.

Of the nine cases clonally related by IG and/or BCL2 breakpoint sequence analysis, all but one (case 1) demonstrated shared mutations between the ISFN and the transformed counterpart(s), ranging from one to six shared mutations per paired samples. For example, the DLBCL of case 4 had four non-synonymous mutations of $B C L 2$, TNFRSF14, HIST1H1D and EP300 in common with the ISFN that was present 159 months prior. However, ISFN and DLBCL of case 5 also exhibited matching KMT2D p.(Q4473*) and IGLL5 p.(C3S) mutations, demonstrating their clonal relationship despite the lack of a detectable clonal IG re-arrangement or $B C L 2$ translocation sequence. All investigated FL showed more than one mutation shared with both the ISFN and the aggressive BCL. Mutations only present in the clinically manifest lymphomas were observed in all cases with the exception of one FL (case 9). Nevertheless, six ISFN lesions also carried private variants that were not identified in their clonally related counterparts, indicating early divergence. The 


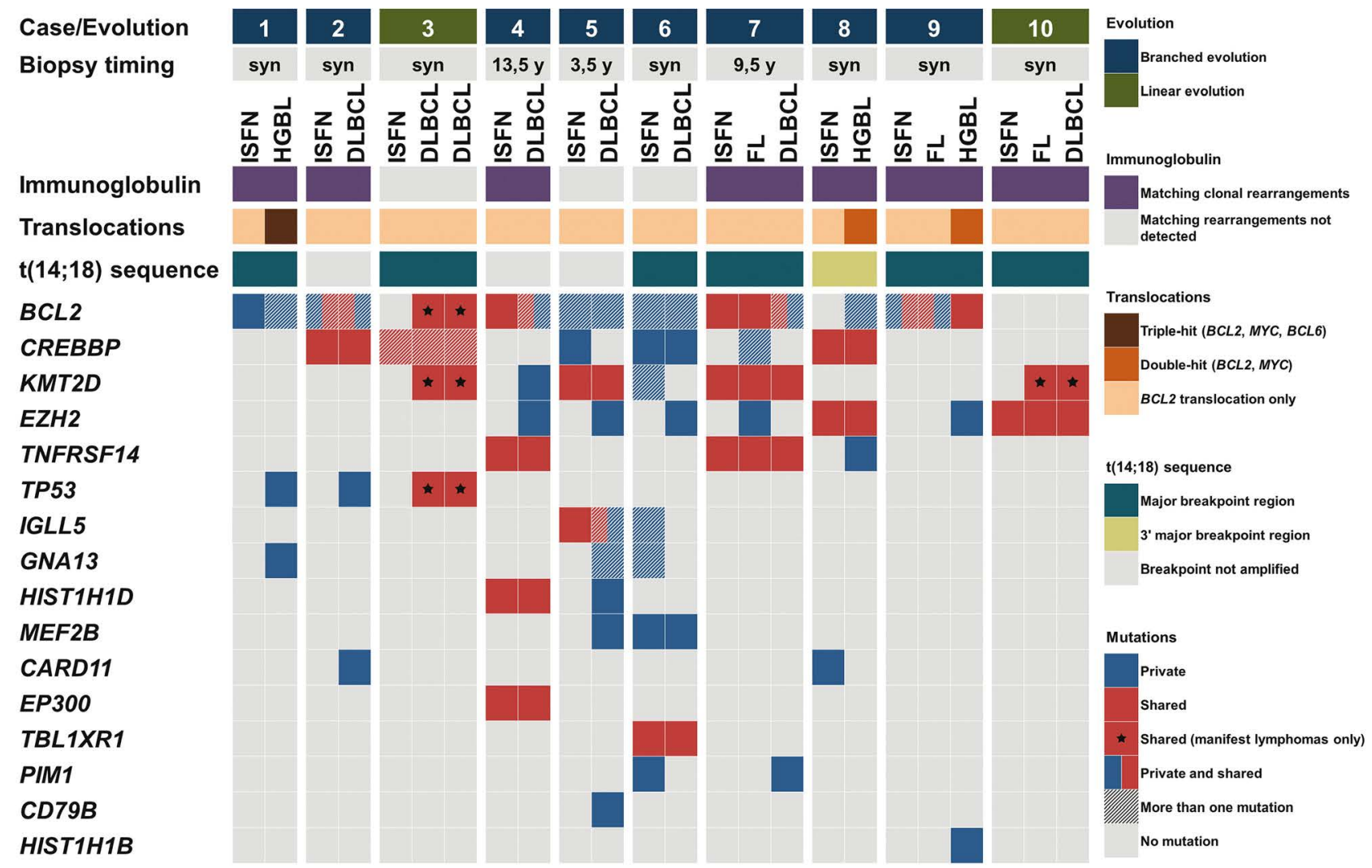

Figure 2. Overview of clonality results, translocations and non-synonymous variants in in situ follicular neoplasia, follicular lymphoma and aggressive B-cell lymphoma samples. Each column of the heatmap represents a sample with paired samples displayed next to each other. Rows represent an analysis or a gene. Genes are ordered from top to bottom according to the mutational frequency across all samples. Biopsy timing specifies the time period between the occurrence of the in situ follicular neoplasia (ISFN) and the diagnosis of aggressive B-cell lymphoma (BCL) in years (" $y$ "), rounded to the nearest half year, with "syn" indicating a synchronous occurrence. In case 4, the assumption of branched evolution is based on a private 5'UTR mutation of BCL2 in the ISFN (mutation not shown). Concerning case 9, "Private and shared mutations" of BCL2 indicate that ISFN and follicular lymphoma (FL) exhibit a shared BCL2 mutation not present in the high-grade B-cell lymphoma while a second BCL2 mutation was shared between all three lesions.

ISFN of case 6 harbored the highest number of private mutations, with a total of 13 different non-synonymous alterations of BCL2, KMT2D, CREBBP, GNA13, MEF2B, PIM1, TBL1XR1, and IGLL5, as well as six synonymous and 5'UTR variants of BCL2, of which only a single TBL1XR1 p.(L198*) mutation was shared with the clonally related aggressive $B C L$.

Based on the distribution of private and shared variants, two different patterns of clonal evolution from ISFN to aggressive BCL could be reconstructed (Figure 3; Online Supplementary Figure S2). The most frequent scenario (cases 1, 2, 4, 5, 6, 7, 8, and 9) was that of branched evolution, where aggressive lymphoma, ISFN and, when present, FL evolved from a common progenitor but gained distinct private mutations (Figure $3 \mathrm{~A}$ and $\mathrm{B}$ ). In contrast, the available data indicate a linear evolution in cases 3 and 10, where the DLBCL shared all ISFN mutations but gained additional alterations (Figure $3 \mathrm{C}$ ).

\section{Discussion}

In this study, we analyzed the clonal evolution of $t(14 ; 18)+$ aggressive BCL from the earliest morphologically identifiable putative precursor lesion - ISFN, using paired samples of ISFN and DLBCL or HGBL with $\mathrm{DH} / \mathrm{TH}$, with and without FL as an intermediate step.
The clonal relationship of ISFN and aggressive BCL samples was confirmed by either identical IGH and/or BCL2 rearrangements and/or the demonstration of shared somatic mutations in genes frequently affected in BCL of GC origin. This study demonstrates for the first time the evolution of "de novo" aggressive BCL from ISFN. Moreover, we identified different pathways of clonal evolution with an early branching pattern (early divergence) as the most frequent scenario.

The progression from ISFN to FL and the transformation of FL to DLBCL or HGBL are well-established. Our study expands these observations and suggests that a direct evolution of $\mathrm{t}(14 ; 18)+$ aggressive BCL from ISFN is possible. This finding is not surprising, given the common presence of discordant (i.e., low-grade) bone marrow infiltration in de novo DLBCL and the occasional recurrence of DLBCL as FL. ${ }^{26-29}$ As for any other neoplasm with a stepwise evolution, we cannot entirely exclude the presence of a clinically and morphologically undetected FL. However, given the fact that approximately $30 \%$ of de novo DLBCL carry a BCL2 translocation, $\mathrm{t}(14 ; 18)+\mathrm{DLBCL}$ arising from ISFN without preceding FL could be a common phenomenon. ${ }^{3,23}$ The more recent analyses of the molecular landscape of DLBCL also support this hypothesis of a shared progenitor population, since $t(14 ; 18)+$ de novo DLBCL revealed a mutational signature very similar to FL..$^{30,31}$ In ISFN and de novo DLBCL of case 5, we were 


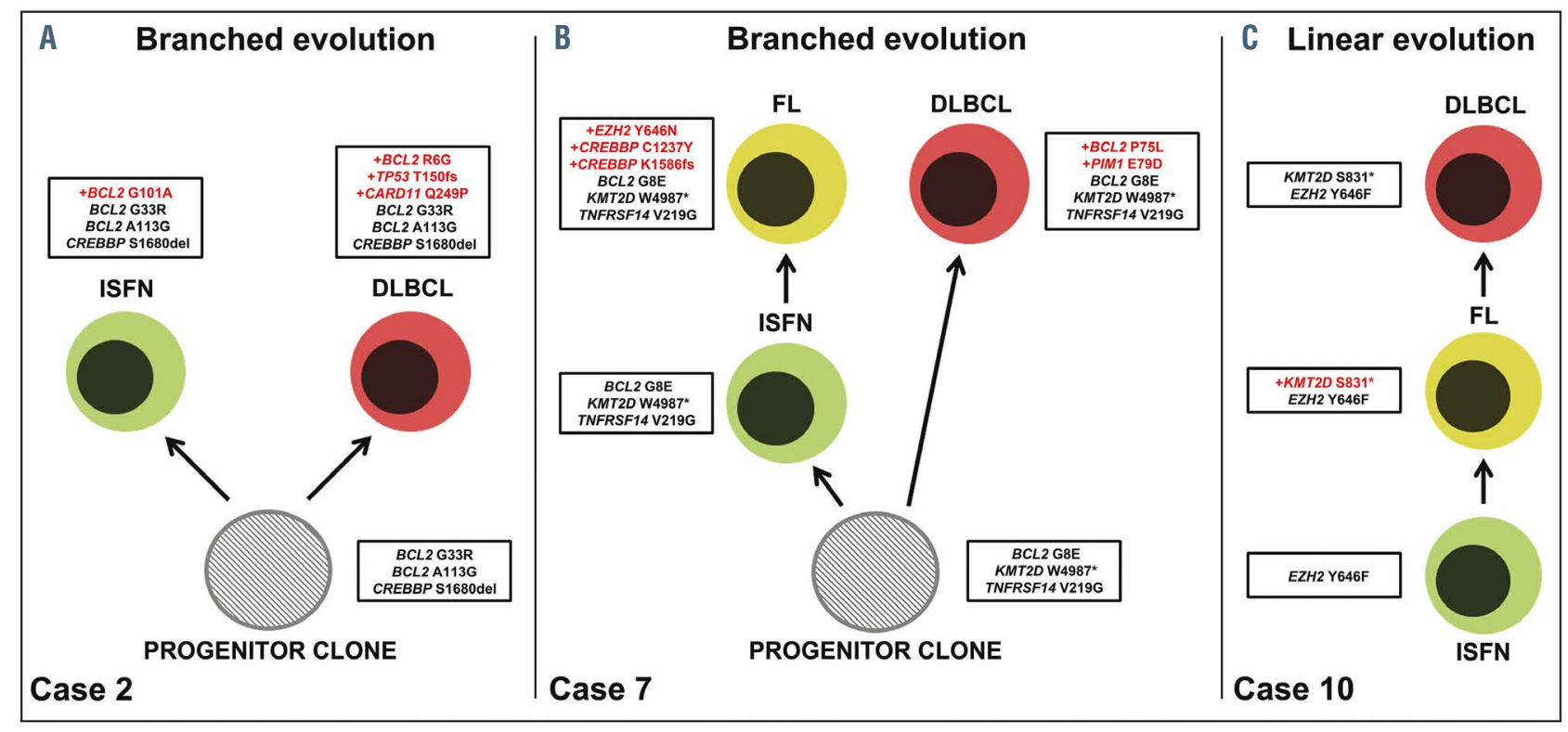

Figure 3. Different patterns of clonal evolution from in situ follicular neoplasia to aggressive B-cell lymphoma based on the distribution of mutations. Variants are depicted at protein level. Mutations highlighted in red were gained during the evolution. Synonymous and 5'UTR variants of BCL2 are not shown, but were also taken into account for the construction. The existence of "Progenitor clones" was assumed on the basis of shared mutations. (A) In situ follicular neoplasia (ISFN) and diffuse large B-cell lymphoma (DLBCL) evolved divergently from a common progenitor clone. (B) ISFN and DLBCL evolved divergently from a common progenitor, with the ISFN subsequently progressing to follicular lymphoma (FL). (C) FL and DLBCL evolved directly from the ISFN and gained additional alterations.

unable to amplify a clonal B-cell rearrangement or the $B C L 2$ translocation sequence. Both samples, however, demonstrated a $B C L 2$ translocation only detectable by FISH using an IGH/BCL2 dual-color, double fusion probe, possibly the result of a cryptic, non-canonical BCL2 rearrangement. ${ }^{32}$ This, in combination with shared mutations of KMT2D and IGLL5, serves as evidence of a common clonal origin.

Clonally related ISFN can be identified not only before or simultaneously with manifest lymphoma, but may also be present even years after the malignant transformation took place, most likely representing a subclone that diverged at an earlier stage of the disease. ${ }^{14,33}$ Persisting precursors, presumably more resistant to chemotherapy, may therefore play a role in lymphoma relapse as well. Indeed, studies of FL and DLBCL relapses have shown that both the primary and the recurrent lymphoma often represent divergent subclones that arose independently from a common progenitor, which again supports our hypothesis that aggressive BCL can develop directly from a premalignant precursor. ${ }^{22,34}$ The existence of such progenitor populations has been exemplified in two reports of clonally related FL and clonally related DLBCL arising in both donor and recipient after hematopoietic stem cell transplantation. $^{35,36}$ In both studies, the related lymphomas exhibited multiple shared alterations, which were therefore acquired prior to transplantation. ${ }^{35,36}$ Likewise, FL and transformed FL have been shown to often arise by divergent evolution. ${ }^{37,38}$ The complexity of this evolutionary process is also reflected in our paired ISFN and aggressive BCL cases, since the distribution of private and shared mutations suggests an early subclonal divergence for the majority of cases. This is supported further when IG data are taken into account, given the high frequency of different glycosylation sites in both components, and the phylogenetic trees based on SHM patterns of rearranged IGH sequences, which are compat- ible with a divergent evolution. Linear evolution from ISFN to aggressive BCL, at least based on the available mutational data, seems to be less frequent, but similar findings have been reported regarding the transformation of FL. ${ }^{38}$ Our IGH data also provide insight into the process of early clonal selection. The more balanced distribution of subclones in the ISFN implies that at this point, no subclone has acquired a decisive selection advantage. In contrast, the aggressive BCL lesions demonstrated one or two highly dominant subclones, which possibly emerged after obtaining crucial secondary genetic alterations that improved clonal fitness and further paved the way towards high-grade malignancy.

The most commonly mutated gene in our study was $B C L 2$, likely because BCL2 juxtaposed to an IG promotor results in a significantly higher number of mutations compared to non-translocated counterparts, as a result of targeting by AID. ${ }^{39,40}$ The abundance of BCL2 mutations across ISFN lesions, as well as the intraclonal heterogeneity revealed by the IGH sequence analysis and the detection of novel $\mathrm{N}$-glycosylation sites confirm that the process of SHM is ongoing in ISFN. ${ }^{15,19}$ Prolonged AID activity is regarded as an important factor in the pathogenesis of GC-derived lymphomas and especially FL. ${ }^{21,22,41}$ In a mouse model, multiple re-entries of long-lived $\mathrm{BCL} 2+\mathrm{B}$-cell clones into the GC environment resulted in the accumulation of secondary alterations with a mutational signature consistent with AID-mediated mutagenesis. ${ }^{42}$ The same concept has been proposed for human FL development, where FLLC are subject to similar dynamics with an extensive dissemination throughout the body, leading to a multitude of subclones exhibiting different SHM signatures as evidence of their GC passage. . $^{17,42}$ Since ISFN is considered the tissue-based counterpart of FLLC, our data also indicate that circulating $\mathrm{t}(14 ; 18)+$ FLLC clones might serve as precursors to aggressive BCL as well. ${ }^{42,43}$ 
The mutational spectrum identified in our study is consistent with published data obtained in FL and $\mathrm{t}(14 ; 18)+$ DLBCL. Both lymphomas were shown to be particularly enriched for mutations in the epigenetic regulators CREBBP, EZH2, KMT2D, and EP300, as well as for alterations of TNFRSF14, a gene encoding for a receptor of the tumor necrosis factor family. $23,30,31,37,38$ CREBBP mutations have consistently been described as drivers of lymphomagenesis and as early genetic events in FL. ${ }^{22,44-47}$ In agreement with this and in line with a recent report, we found CREBBP to be the most commonly affected gene in ISFN samples, with mutations being shared with the clonally related manifest lymphomas in three cases. ${ }^{15}$ Moreover, ISFN and DLBCL of case 4, which lacked CREBBP alterations, exhibited a shared mutation of the closely related acetyltransferase EP300. CREBBP and EP300 mutations have been suggested to play similar roles in the pathogenesis of FL and DLBCL and are therefore usually mutually exclusive. $^{23,47,48}$ Alterations of EZH2, KMT2D, and TNFRSF14 have been described as both early driver and as accelerator mutations..$^{37,38,44-46,49}$ We confirm that these mutations can occur during the presumably earliest stages of the disease, evidently years before the diagnosis of malignant lymphoma. ${ }^{14,15}$ Alterations of KMT2D and EZH2 were, however, detected only in the manifest lymphomas of three of and five of seven cases respectively, suggesting they were often acquired later, possibly driving the clone towards the malignant transformation. Notably, BCL2 mutations frequently occur at the earliest stages as well and are likely primarily an indicator of AID activity, rather than heralding aggressive behavior, as suggested by other authors. ${ }^{50}$ PIM1 and IGLL5, two additional genes known to be affected by aberrant SHM, were also mutated in one and two ISFN samples, respectively. ${ }^{31}$ Re-arrangements of MYC and alterations of TP53 are common drivers of FL transformation. ${ }^{38}$ Accordingly, five of our cases carried these genetic alterations and as expected, they were only detected in the aggressive BCL and not in the clonally related ISFN lesions. However, due to technical limitations, the presence of these mutations in minor ISFN subclones cannot be excluded completely.

Although we were able to investigate the evolution of ISFN at multiple levels, this study has some limitations, in particular, the small sample size due to the rarity of identifiable ISFN lesions in patients with aggressive BCL, which warrants validation in further studies. The necessary restriction to FFPE tissue also narrowed the scope of feasible analyses and raised the detection threshold in our targeted NGS analysis because of low level sequencing artifacts. Nevertheless, systematic validation allowed us to delineate the clonal and genetic evolution of aggressive BCL starting from an early progenitor lesion.

In summary, our data extend previous studies and provide first evidence that $t(14 ; 18)+$ DLBCL and HGBL can arise from clonally related ISFN without FL as an intermediate step. Moreover, during this progression, similar to the clonal evolution and transformation of $\mathrm{FL}$, branched evolution with both private and shared alterations is common. Our results further confirm that ISFN is subject to persistent AID activity and frequently acquires secondary genetic alterations, in addition to the defining $t(14 ; 18)$ translocation.

\section{Disclosures}

No conflicts of interest to disclose.

\section{Contributions}

$F F, I B$ and $A V$ wrote the manuscript; FF conceived and designed the study, selected the cases, and supervised the experimental work and data analysis; LQ- $M$ helped designing the study, reviewed the cases and helped writing the manuscript; $A V$ performed the experimental work and analyzed the data; JuS, $J a S$, and IB supervised the experimental work and data analysis; BM performed FISH analysis; BF and IAM-M helped with case selection; $P B$ and $S N$ provided bioinformatics support and constructed the phylogenetic trees; MR-P, MAP, KH and GO contributed with cases and provided clinical information. All contributing authors revised the manuscript.

\section{Acknowledgements}

The authors would like to thank Julia Bein, Sylvia Hartmann and Martin-Leo Hansmann for their cooperation and are grateful to Franziska Mihalik, Rebecca Braun, Inga Müller, Dennis Thiele, Isabell Haußmann, Gerd Janke, and Sema Colak for their excellent technical assistance. We acknowledge support by Open Access Publishing Fund of University of Tübingen.

\section{Funding}

This work was supported by grants from the Deutsche Forschungsgemeinschaft (DFG) to LQ-M (QU144/1-1) and FF (FE597/4-1) and the IZKF Promotionskolleg (E0500520).

\section{References}

1. Alizadeh AA, Eisen MB, Davis RE, et al. Distinct types of diffuse large B-cell lymphoma identified by gene expression profiling. Nature. 2000;403(6769):503-511

2. Hans CP, Weisenburger DD, Greiner TC, et al. Confirmation of the molecular classification of diffuse large B-cell lymphoma by immunohistochemistry using a tissue microarray. Blood. 2004;103(1):275-282

3. Swerdlow SH, Campo E, Harris NL, et al. WHO Classification of Tumours of Haematopoietic and Lymphoid Tissues. Revised 4th ed. Lyon: IARC Press. 2017.

4. Tsujimoto Y, Gorham J, Cossman J, Jaffe E, Croce CM. The $t(14 ; 18)$ chromosome translocations involved in B-cell neoplasms result from mistakes in VDJ joining. Science. 1985;229(4720):1390-1393.

5. McDonnell TJ, Deane N, Platt FM, et al. bcl2 -immunoglobulin transgenic mice demon- strate extended B cell survival and follicular lymphoproliferation. Cell. 1989;57(1):79-88.

6. Smith KG, Light A, O'Reilly LA, Ang SM, Strasser A, Tarlinton D. bcl-2 transgene expression inhibits apoptosis in the germinal center and reveals differences in the selection of memory B cells and bone marrow antibody-forming cells. J Exp Med. 2000;191(3):475-484.

7. Kridel R, Mottok A, Farinha P, et al. Cell of origin of transformed follicular lymphoma. Blood. 2015;126(18):2118-2127.

8. Dölken G, Dölken L, Hirt C, Fusch C, Rabkin CS, Schüler F. Age-dependent prevalence and frequency of circulating $t(14 ; 18)$ positive cells in the peripheral blood of healthy individuals. J Natl Cancer Inst Monogr. 2008;(39):44-47.

9. Roulland S, Lebailly P, Lecluse Y, Heutte N, Nadel B, Gauduchon P. Long-term clonal persistence and evolution of $\mathrm{t}(14 ; 18)$-bearing $\mathrm{B}$ cells in healthy individuals. Leukemia.
2006;20(1):158-162

10. Roulland S, Kelly RS, Morgado E, et al $t(14 ; 18)$ Translocation: a predictive blood biomarker for follicular lymphoma. J Clin Oncol. 2014;32(13):1347-1355.

11. Cong P, Raffeld M, Teruya-Feldstein J, Sorbara L, Pittaluga S, Jaffe ES. In situ localization of follicular lymphoma: description and analysis by laser capture microdissection. Blood. 2002;99(9):3376-3382

12. Jegalian AG, Eberle FC, Pack SD, et al Follicular lymphoma in situ: clinical implications and comparisons with partial involvement by follicular lymphoma. Blood. 2011;118(11):2976-2984.

13. Henopp T, Quintanilla-Martinez L, Fend F, Adam P. Prevalence of follicular lymphoma in situ in consecutively analysed reactive lymph nodes. Histopathology. 2011;59(1): 139-142.

14. Schmidt J, Salaverria I, Haake A, et al. Increasing genomic and epigenomic com- 
plexity in the clonal evolution from in situ to manifest $\mathrm{t}(14 ; 18)$-positive follicular lymphoma. Leukemia. 2014;28(5):1103-1112.

15. Schmidt J, Ramis-Zaldivar JE, Bonzheim I, et al. CREBBP gene mutations are frequently detected in in situ follicular neoplasia. Blood. 2018;132(25):2687-2690.

16. Mamessier E, Song JY, Eberle FC, et al. Early lesions of follicular lymphoma: a genetic perspective. Haematologica. 2014;99(3):481488.

17. Agopian J, Navarro JM, Gac AC, et al. Agricultural pesticide exposure and the molecular connection to lymphomagenesis. J Exp Med. 2009;206(7):1473-1483.

18. Mamessier E, Drevet C, BroussaisGuillaumot F, et al. Contiguous follicular lymphoma and follicular lymphoma in situ harboring N-glycosylated sites. Haematologica. 2015;100(4): e155-157.

19. Kosmidis P, Bonzheim I, Dufke C, et al. Next generation sequencing of the clonal IGH rearrangement detects ongoing mutations and interfollicular trafficking in in situ follicular neoplasia. PLoS One. 2017;12(6): e0178503.

20. Coelho V, Krysov S, Ghaemmaghami AM, et al. Glycosylation of surface Ig creates a functional bridge between human follicular lymphoma and microenvironmental lectins. Proc Natl Acad Sci U S A. 2010;107 (43):18587-18592.

21. Greeve J, Philipsen A, Krause K, et al. Expression of activation-induced cytidine deaminase in human B-cell non-Hodgkin lymphomas. Blood. 2003;101(9):3574-3580.

22. Loeffler M, Kreuz M, Haake A, et al. Genomic and epigenomic co-evolution in follicular lymphomas. Leukemia. 2015;29(2):456-463.

23. Morin RD, Mendez-Lago M, Mungall AJ, et al. Frequent mutation of histone-modifying genes in non-Hodgkin lymphoma. Nature. 2011:476(7360):298-303

24. Aukema SM, Kreuz M, Kohler CW, et al. Biological characterization of adult MYCtranslocation-positive mature B-cell lymphomas other than molecular Burkitt lymphoma. Haematologica. 2014;99(4):726-735.

25. van Dongen JJ, Langerak AW, Bruggemann $M$, et al. Design and standardization of PCR primers and protocols for detection of clonal immunoglobulin and T-cell receptor gene recombinations in suspect lymphoproliferations: report of the BIOMED-2 Concerted Action BMH4-CT98-3936. Leukemia. 2003;17(12):2257-2317.

26. Lee AY, Connors JM, Klimo P, O'Reilly SE,
Gascoyne RD. Late relapse in patients with diffuse large-cell lymphoma treated with MACOP-B. J Clin Oncol. 1997;15(5):1745 1753.

27. Arber DA, George TI. Bone marrow biopsy involvement by non-Hodgkin's lymphoma: frequency of lymphoma types, patterns, blood involvement, and discordance with other sites in 450 specimens. Am J Surg Pathol. 2005;29(12):1549-1557.

28. Brudno I, Tadmor T, Pittaluga S, Nicolae A Polliack A, Dunleavy K. Discordant bone marrow involvement in non-Hodgkin lymphoma. Blood. 2016:127(8):965-970.

29. Kremer M, Spitzer M, Mandl-Weber S, et al. Discordant bone marrow involvement in diffuse large B-cell lymphoma: comparative molecular analysis reveals a heterogeneous group of disorders. Lab Invest. 2003;83(1): $107-114$.

30. Schmitz R, Wright GW, Huang DW, et al. Genetics and pathogenesis of diffuse large $B$ cell lymphoma. N Engl J Med. 2018;378 (15):1396-1407.

31. Chapuy B, Stewart C, Dunford AJ, et al Molecular subtypes of diffuse large B cell lymphoma are associated with distinct pathogenic mechanisms and outcomes. Nat Med. 2018;24(5):679-690.

32. Yamamoto K, Okamura A, Inui $Y$, et al. Cryptic insertion of BCL2 gene into immunoglobulin heavy locus in follicula lymphoma with $\mathrm{t}(6 ; 9)(\mathrm{p} 23 ; \mathrm{p} 13)$. Leuk Res. 2012;36(9):e202-205.

33. Bonzheim I, Salaverria I, Haake A, et al. A unique case of follicular lymphoma provides insights to the clonal evolution from follicular lymphoma in situ to manifest follicular lymphoma. Blood. 2011;118(12):3442-3444.

34. Juskevicius D, Lorber T, Gsponer J, et al. Distinct genetic evolution patterns of relapsing diffuse large B-cell lymphoma revealed by genome-wide copy number aberration and targeted sequencing analysis. Leukemia. 2016;30(12):2385-2395

35. Weigert O, Kopp N, Lane AA, et al Molecular ontogeny of donor-derived follicular lymphomas occurring after hematopoietic cell transplantation. Cancer Discov. 2012;2(1): 47-55

36. Araf S, Wang J, Ashton-Key $\mathrm{M}$, et al. Transmission of diffuse large B-cell lymphoma by an allogeneic stem-cell transplant. Haematologica. 2019;104(4):e174-e177.

37. Okosun J, Bodor C, Wang J, et al. Integrated genomic analysis identifies recurrent mutations and evolution patterns driving the initiation and progression of follicular lym- phoma. Nat Genet. 2014;46(2):176-181.

38. Pasqualucci L, Khiabanian H, Fangazio M, et al. Genetics of follicular lymphoma transformation. Cell Rep. 2014;6(1):130-140.

39. Saito M, Novak U, Piovan E, et al. BCL6 suppression of BCL2 via Miz1 and its disruption in diffuse large B cell lymphoma. Proc Nat Acad Sci U S A. 2009:106(27):11294-11299.

40. Schuetz JM, Johnson NA, Morin RD, et al. BCL2 mutations in diffuse large B-cell lymphoma. Leukemia. 2012;26(6):1383-1390.

41. Pasqualucci L, Bhagat $G$, Jankovic $M$, et al. AID is required for germinal center-derived lymphomagenesis. Nat Genet. 2008;40(1): 108-112.

42. Sungalee S, Mamessier E, Morgado E, et al. Germinal center reentries of BCL2-overexpressing B cells drive follicular lymphoma progression. J Clin Invest. 2014;124(12) 5337-5351

43. Cheung MC, Bailey D, Pennell N, et al. In situ localization of follicular lymphoma: evidence for subclinical systemic disease with detection of an identical BCL-2/IGH fusion gene in blood and lymph node. Leukemia. 2009:23(6):1176-1179.

44. Green MR, Gentles AJ, Nair RV, et al. Hierarchy in somatic mutations arising during genomic evolution and progression of follicular lymphoma. Blood. 2013;121(9): 1604-1611.

45. Green MR, Kihira S, Liu CL, et al. Mutations in early follicular lymphoma progenitors are associated with suppressed antigen presentation. Proc Natl Acad Sci U S A. 2015;112 (10):E1116-1125.

46. Kridel R, Chan FC, Mottok A, et al Histological transformation and progression in follicular lymphoma: a clonal evolution study. PLoS Med. 2016;13(12):e1002197.

47. Pasqualucci L, Dominguez-Sola D Chiarenza A, et al. Inactivating mutations of acetyltransferase genes in B-cell lymphoma. Nature. 2011:471(7337):189-195.

48. Zhang J, Vlasevska S, Wells VA, et al. The CREBBP acetyltransferase is a haploinsufficient tumor suppressor in B-cell lymphoma. Cancer Discov. 2017;7(3):322-337.

49. Jiang Y, Redmond D, Nie K, et al. Deep sequencing reveals clonal evolution patterns and mutation events associated with relapse in B-cell lymphomas. Genome Biol 2014:15(8):432.

50. Correia C, Schneider PA, Dai H, et al. BCL2 mutations are associated with increased risk of transformation and shortened survival in follicular lymphoma. Blood. 2015;125(4): 658-667. 